\title{
A Soluble Flavonoid-glycoside, $\alpha$ G-Rutin, Is Absorbed as Glycosides in the Isolated Gastric and Intestinal Mucosa
}

\author{
Megumi Matsumoto, ${ }^{1,2}$ Noriko Matsukawa, ${ }^{2}$ Hitoshi Mineo, ${ }^{2}$ \\ Hideyuki CHIJ, ${ }^{2}$ and Hiroshi HARA ${ }^{1, \dagger}$ \\ ${ }^{1}$ Laboratory of Nutritional Biochemistry, Division of Applied Bioscience, Graduate School of Agriculture, \\ Hokkaido University, Sapporo, Hokkaido 060-8589, Japan \\ ${ }^{2}$ Department of Food Science and Human Nutrition, Faculty of Human Life Science, Fuji Women's University, \\ Ishikari, Hokkaido 061-3204, Japan
}

Received April 22, 2004; Accepted July 1, 2004

\begin{abstract}
We investigated the absorption and metabolism of the highly soluble quercetin glycoside $\alpha \mathrm{G}$-rutin, a glucose adduct of insoluble rutin, using the isolated mucosa of the rat stomach and intestines equipped with the Ussing chamber. $\alpha$ G-rutin and rutin appeared in the serosal sides of the gastric body and all the intestinal mucosa after the addition of $\alpha \mathrm{G}$-rutin ( $1 \mathrm{~mm}$ ) to the mucosal fluid. The degree of $\alpha \mathrm{G}$-rutin appearance was much lower in the gastric fundus than in the other parts. Quercetin was not found in the mucosal fluid of any mucosal specimen. The concentrations $(\mu \mathrm{M})$ of $\alpha \mathrm{G}$-rutin and rutin in the serosal fluid as a result of transport from the mucosal side increased time-dependently and linearly with mucosal $\alpha$ G-rutin concentration $(1,10$ or $100 \mathrm{~mm})$. The highest transport was shown in the ileal mucosa. These results indicate that $\alpha \mathrm{G}$-rutin is partly hydrolyzed to rutin through the intestine and absorbed as such.
\end{abstract}

Key words: $\alpha$ G-rutin; flavonoid-glycoside; absorption; Ussing chamber; gastrointestine

Polyphenols are widely distributed in the plant world, especially in fruits and vegetables, as secondary metabolites. ${ }^{1)}$ These compounds act as antioxidants both in foods and in the body. ${ }^{2}$ Flavonoids, a kind of polyphenol, are ingested as natural food pigments or supplements, however, the absorption and metabolism of flavonoids, and especially of their glycosides, are not yet fully understood. The bioavailability of these compounds depends on intestinal absorption, which is defined by their chemical composition. Quercetin is a well-known natural flavonoid found in onions, green tea, and sophora. ${ }^{3-5)}$ It is present mainly as $\beta$-glycosides in plant foods. The nature of glycosylation probably influences the efficiency of quercetin absorption. ${ }^{6}$ Quercetin-3-O-glucoside does not appear in systemic blood after perfusion of this quercetin-glycoside to the rat small intestine. It is suggested that hydrolysis by mucosal lactase phlorizin hydrolase (LPH) is a major step in the intestinal absorption of quercetin-glucoside. ${ }^{7)}$ Another report suggests that a glucose transporter (SGLT1) may facilitate the transport of quercetin-3-Oglucoside in the isolated mucosa of the rat jejunum and proximal colon, ${ }^{8)}$ but there is very little information about the intestinal absorption of other flavonoid glycosides.

Recently, a new quercetin glycoside, $\alpha$ G-rutin, has been developed and made available (Fig. 1). This glycoside is a highly water-soluble quercetin derivative, in which one D-glucose is attached to rutin (quercetin-3$O$-glucosyl-rhamnose). Previous in vivo experiments have shown that rutin is neither readily absorbed nor metabolized in the small intestine. ${ }^{6,9,10)}$ It is hardly soluble in water or other solvents, which may be one reason for its low absorbability in the small intestine. ${ }^{10-12)}$ Rutin absorption might be increased by improvement of water-solubility. Nonetheless, the intestinal absorption of $\alpha$ G-rutin has not yet been evaluated.

The aim of the present study is to investigate the absorption and metabolism of the quercetin glycoside

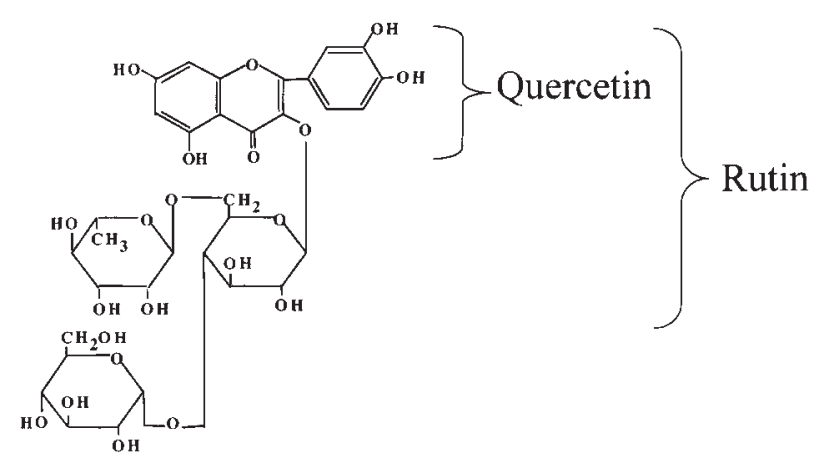

Fig. 1. Structure of $\alpha \mathrm{G}$-Rutin.

\footnotetext{
$\dagger$ To whom correspondence should be addressed. Tel: +81-11-706-2504; Fax: +81-11-706-3352; E-mail: hara@ @hem.agr.hokudai.ac.jp
} 
( $\alpha$ G-rutin) in the rat stomach and intestines. We examined its absorption and metabolism by the isolated mucosa of the stomach, small intestine, cecum, and colon with an Ussing chamber. $\alpha$ G-rutin and its metabolites were quantified by LC/MS analysis.

\section{Materials and Methods}

Chemicals. $\alpha$ G-rutin was provided by Toyo Sugar Refining (Tokyo, Japan). Quercetin and rutin were obtained from Wako Pure Chemical Industries (Osaka, Japan). All other reagents and chemicals commercially available were of extra-pure grade.

Animals and diets. Male Wistar/ST rats (Japan SLC, Sizuoka, Japan) weighing 230-250g were housed in individual cages in a temperature-controlled room at $22^{\circ} \mathrm{C}$ throughout the experimental period. The rats were fed a $25 \%$ casein-sucrose diet consisting of $60.25 \%$ sucrose, $25 \%$ casein, $5 \%$ cellulose, $3.5 \%$ mineral mixture (AIN 93G), 1\% vitamin mixture (AIN 93G), $5 \%$ corn oil, and $0.25 \%$ choline chloride for a week just before use.

These studies were approved by the Hokkaido University Animal Committee. The animals were maintained in accordance with Hokkaido University guidelines for the care and use of laboratory animals.

Absorption and metabolism by isolated gastro-intestinal mucosa. The stomach, small intestine (from the ligament of Treitz to the ileocecal junction), cecum, and colon were removed from six rats under anesthesia with pentobarbital $(40 \mathrm{mg} / \mathrm{kg}$ body weight, Nembutal, Abbott, North Chicago, Illinois, U.S.A.). The outside and inside surfaces of the isolated segments were washed with ice-cold saline $(154 \mathrm{~mm} \mathrm{NaCl})$. The excised small intestine was flushed with saline $(20 \mathrm{ml})$. The stomach (whole sac), jejunum $(15 \mathrm{~cm}$ distal from the Trietz ligament), ileum $(15 \mathrm{~cm}$ proximal from the ileocecal junction), cecum (whole sac), and colon (middle and distal parts, each one third of the whole colon) segments were cut open along the mesenteric border to be a flat sheet and rinsed with an ice-cold balanced salt solution buffered by HEPES (HBS): $125 \mathrm{mM} \mathrm{NaCl}, 4 \mathrm{mM} \mathrm{KCl}$, $10 \mathrm{~mm}$ D-glucose, $30 \mathrm{~mm}$ HEPES, and $1.25 \mathrm{mM} \mathrm{CaCl}_{2}$, gassed with $100 \% \mathrm{O}_{2}, \mathrm{pH} 7.4$. The stomach was divided into two parts, the upper stomach (gastric fundus) and the lower stomach (gastric body). The serosa and muscle layers of the segments were removed, and preparations consisting of the mucosal and submucosal tissues were mounted onto Ussing chambers (diffusion chamber system, Corning Costar, Cambridge, U.K.) which exposed a circular area of the epithelium $\left(0.64 \mathrm{~cm}^{2}\right)$ to the medium. The serosal and mucosal sides of the specimens were bathed in $1 \mathrm{ml}$ of HBS continuously gassed by $100 \% \mathrm{O}_{2}$. After a 30 -min stabilization period, the serosal medium was replaced with fresh HBS, and the mucosal medium was replaced with 1,10 , or $100 \mathrm{~mm}$ $\alpha$ G-rutin in HBS. After incubation for 30 or $60 \mathrm{~min}$ at $37^{\circ} \mathrm{C}$, the serosal solution was collected.

The integrity and viability of the tissue was checked by transepithelial electrical resistance (TEER, MillicellERS, Millipore, Billerica, Massachusetts, U.S.A.). The TEER value of the epithelial preparation in the gastric fundus, gastric body, jejunum, ileum, cecum, and colon was measured before and after the 30-min incubation period with $100 \mathrm{~mm} \alpha$ G-rutin added to the mucosal chamber. The TEER value was indicated in terms of $\Omega \mathrm{cm}^{-2}$.

LC/MS analysis. The serosal fluid samples $(100 \mu \mathrm{l})$ were added to $100 \mu \mathrm{l}$ of $\mathrm{MeOH}$, heated at $100^{\circ} \mathrm{C}$ for $1 \mathrm{~min}$, and centrifuged for $3 \mathrm{~min}$ at $9,000 \times g$. The supernatant was applied to oasis HLB cartridges (Waters, Milford, Massachusetts, U.S.A.), and the eluent was dried and dissolved in $100 \mu \mathrm{l}$ of $50 \% \mathrm{MeOH}$ solution (sample solution).

Quercetin derivatives were identified and quantified with a Waters mass spectrometer-computer system through the positive ions at the electric spray ionization (ESI)-interface (ZQ 2000, Waters). The temperature of the capillary heater and the vaporization heater was maintained at $100{ }^{\circ} \mathrm{C}$ and $300{ }^{\circ} \mathrm{C}$ respectively. The flow rate of sheath gas (nitrogen) was 70 arb. LC/ESI-MS was carried out in the scan mode from $m / z: 50$ to 2,000 $\left(\mathrm{M}^{+}\right)$and in the selected ion monitoring (SIM) mode $m / z: 303\left(\mathrm{M}^{+}\right)$for quercetin, $m / z: 611\left(\mathrm{M}^{+}\right)$for rutin, and $m / z: 773\left(\mathbf{M}^{+}\right)$for $\alpha \mathrm{G}$-rutin respectively. The HPLC system was fitted with a 5- $\mu \mathrm{m}$ C-18 Waters Puresil TM column $(150 \mathrm{~mm} \times 4.6 \mathrm{~mm}$, Waters $)$, and the temperature was maintained by a column oven set at $40^{\circ} \mathrm{C}$. Solvents A (water:methanol:trifluoroacetic acid, 70:30: $0.1, \mathrm{v} / \mathrm{v} / \mathrm{v}$ ) and $\mathrm{B}$ (methanol:trifluoroacetic acid, 100: $0.1, \mathrm{v} / \mathrm{v}$ ), were run at a flow rate of $1 \mathrm{ml} / \mathrm{min}$ using a linear gradient up to $30 \%$ from $10 \%$ of solvent B until 20 min and back to $10 \%$ solvent $\mathrm{B}$ for the next 5 min, and then held in this condition for a further $5 \mathrm{~min}$. Concentrations of flavonoids in the sample solution were estimated using the calibration curves of quercetin, rutin, and $\alpha \mathrm{G}$-rutin standard solution. UV chromatograms were also recorded at $360 \mathrm{~nm}$.

Calculations. Statistical analyses were performed by one-way and two-way ANOVA. The differences among groups were analyzed by Duncan's multiple range test and were considered significant at $P<0.05$.

\section{Results}

The LC/MS chromatograms of $\alpha \mathrm{G}$-rutin, quercetin, and rutin in the standard solution (A) and sample solution (B) are shown in Fig. 2. The retention times for $\alpha \mathrm{G}$-rutin and rutin were $7 \mathrm{~min}$, and that of quercetin was $15 \mathrm{~min}$. Intact $\alpha \mathrm{G}$-rutin and rutin were detected chromatographically by absorbance measurement at $360 \mathrm{~nm}$ in the serosal fluid $30 \mathrm{~min}$ after incubation, with the 

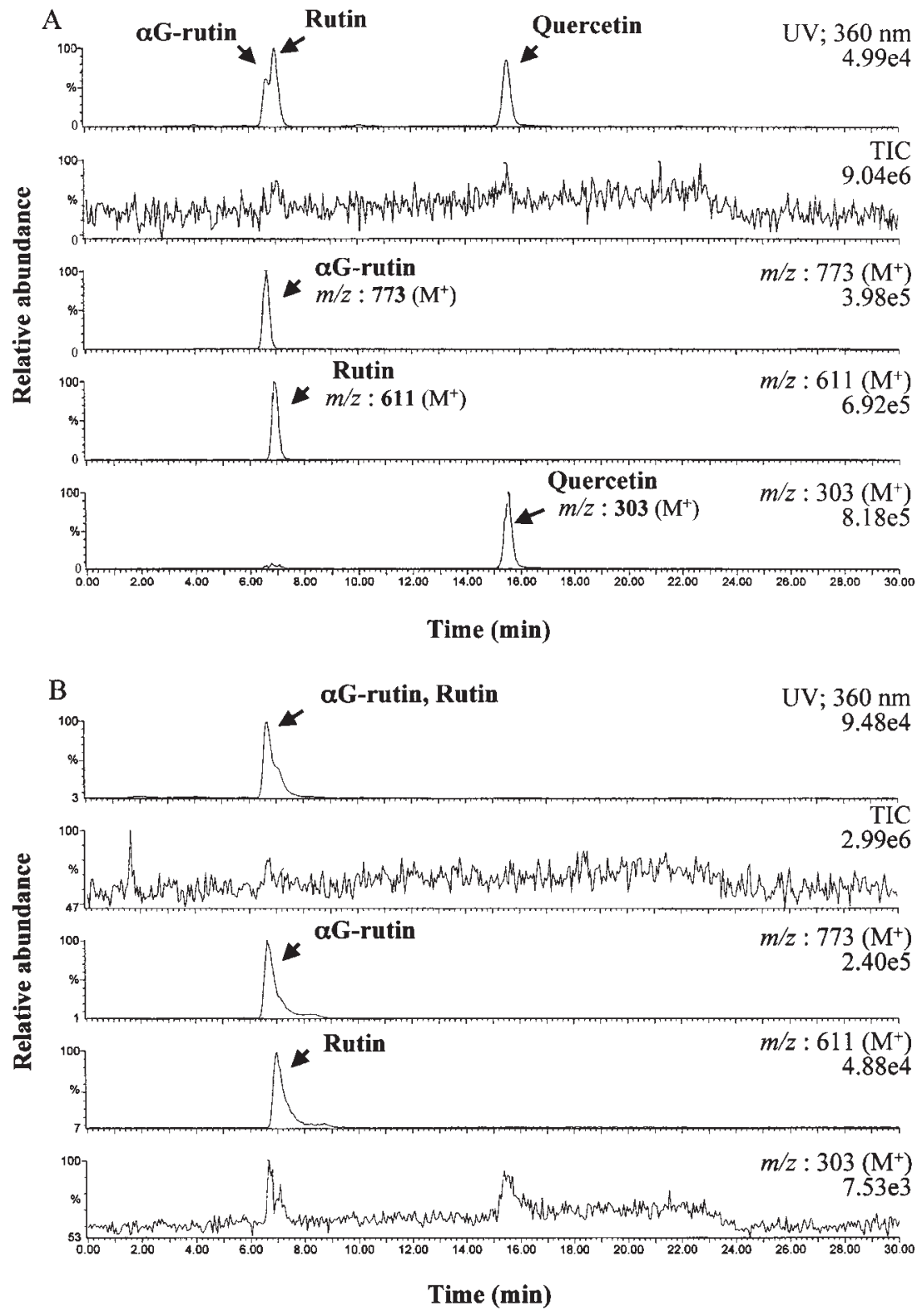

Fig. 2. LC/MS Analysis of Standard of $\alpha$ G-Rutin, Rutin, and Quercetin (A), $\alpha$ G-Rutin and Rutin in the Serosal Fluid of the Ileum after Incubation for $30 \mathrm{~min}(\mathrm{~B})$.

LC/MS chromatogram monitoring relative absorption at $360 \mathrm{~nm}$ and selected ion monitoring (SIM) mode $m / z: 303\left(\mathrm{M}^{+}\right)$for quercetin, $m / z$ : $611\left(\mathrm{M}^{+}\right)$for rutin, and $m / z: 773\left(\mathrm{M}^{+}\right)$for $\alpha \mathrm{G}$-rutin.

mucosal fluid containing $1 \mathrm{~mm}$ of $\alpha \mathrm{G}$-rutin (Fig. 2B).

$\alpha \mathrm{G}$-rutin and rutin concentrations in the gastric fundus, gastric body, jejunum, ileum, cecum, and colon increased time-dependently from $30 \mathrm{~min}$ to $60 \mathrm{~min}$. (Fig. 3). The highest concentrations of $\alpha$ G-rutin and rutin were found in the ileal serosal fluid at $60 \mathrm{~min}$, and was $0.03 \%$ of total $\alpha \mathrm{G}$-rutin in the mucosal fluid.

$\alpha \mathrm{G}$-rutin and rutin in the serosal fluid of the mucosal specimens of the gastric fundus, gastric body, jejunum, ileum, cecum, and colon dose-dependently increased with increased $\alpha$ G-rutin $(1,10$, or $100 \mathrm{~mm}$ ) in the mucosal fluid (fluid, see Fig. 4). Increases in rutin in the serosal fluid were comparable to those of $\alpha$ G-rutin in the ileal mucosa up to $10 \mathrm{~mm} \alpha \mathrm{G}$-rutin in the mucosal fluid, but the rutin concentration was much lower than that of $\alpha$ G-rutin at $100 \mathrm{~mm}$ in both the mucosa. The concentrations of rutin in the serosal fluid of the cecum and colon were very low at all the concentrations of mucosal $\alpha \mathrm{G}$-rutin. The concentrations in the gastric fundus were much lower than in the other parts of the mucosa. The transport rates of $\alpha \mathrm{G}$-rutin and rutin into the serosal fluid with $100 \mathrm{~mm} \alpha \mathrm{G}$-rutin in the mucosal fluid were 0.14 and 0.01 in the gastric fundus, 0.49 and 0.17 in the gastric body, 2.04 and 0.39 in the jejunum, 2.90 and 0.68 in the ileum, 3.83 and 0.20 in the cecum, and 0.95 and $0.01 \mathrm{nmol} / \mathrm{min} / \mathrm{cm}^{2}$ in the colon respectively. Quercetin and quercetin conjugates were not detected in the serosal fluid. 

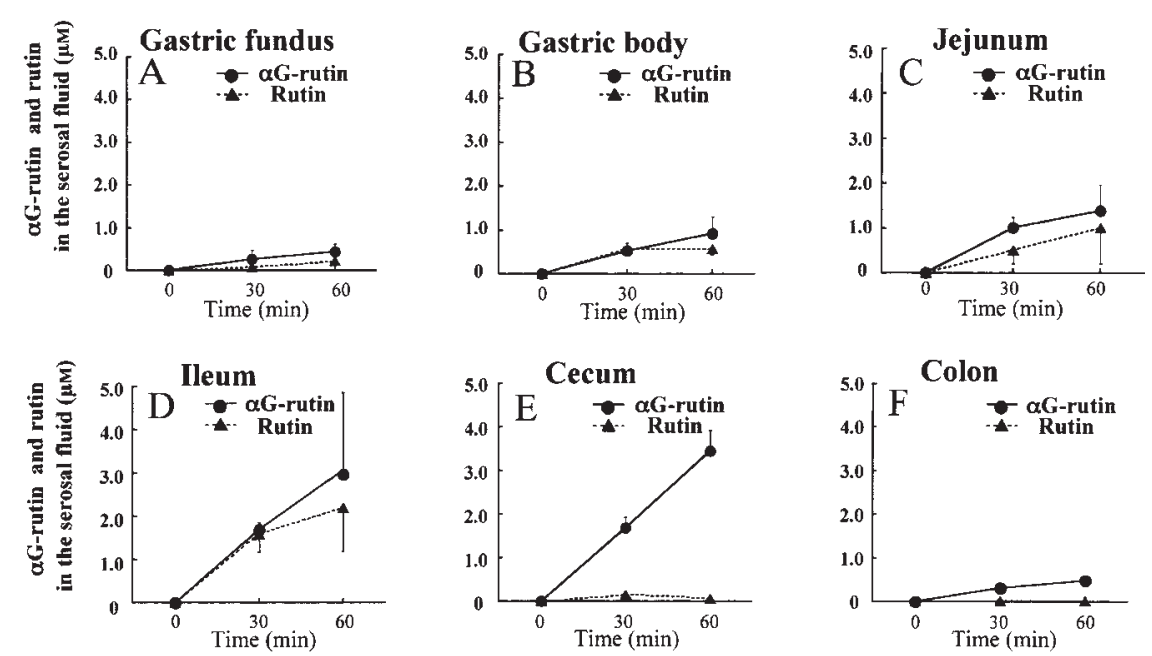

Fig. 3. Concentrations of $\alpha$ G-Rutin and Rutin in the Isolated Stomach Fundus (A), Stomach Body (B), Jejunum (C), Ileum (D), Cecum (E), and Colon (F) of Rats after Incubation for $30 \mathrm{~min}$ or $60 \mathrm{~min}$ at $37^{\circ} \mathrm{C}$.

HBS without $\alpha$ G-rutin was applied to the serosal bath and $1 \mathrm{~mm} \alpha \mathrm{G}$-rutin-HBS was applied to the mucosal bath. Values are means \pm SEM, $\mathrm{n}=3$.

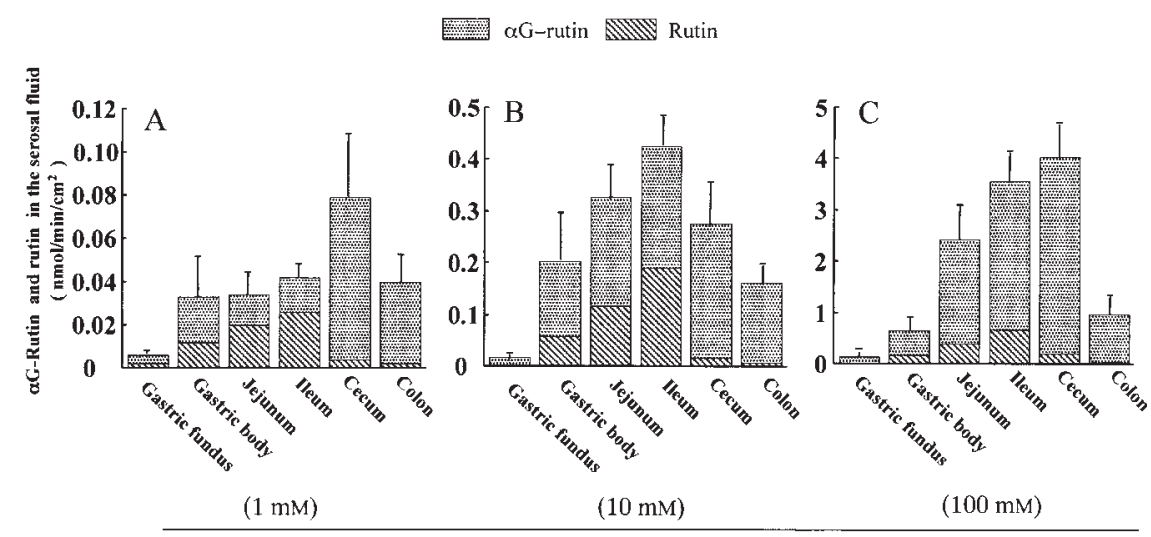

$\alpha G-R u t i n$ in the mucosal fluid

Fig. 4. Dose-dependent Transportation of $\alpha \mathrm{G}$-Rutin in the Isolated Small Intestinal and Cecal Mucosa.

HBS containing 1 (A), 10 (B), or 100 (C) $\mathrm{mM} \alpha$ G-rutin was applied to the mucosal bath and incubated for 30 min at $37^{\circ} \mathrm{C}$. Values are means \pm SEM, $\mathrm{n}=7$.

The TEER in the six intestinal portions before and after incubation for $30 \mathrm{~min}$ are shown in Table 1 . The 30-min incubation with $100 \mathrm{~mm} \alpha$ G-rutin did not affect the TEER for any portion of the intestine. The values in the mucosa of the gastric fundus were more than 10 times those of the other parts.

\section{Discussion}

In this study, we examined the absorption and metabolism of a D-glucose adduct of rutin, $\alpha$ G-rutin, using the isolated mucosa of the rat stomach and intestines. The serosa layers of the isolated mucosa were removed in the epithelial preparation used. Hence, as compared with an everted intestine sac, the ability to transport a substance could be measured more sensitively and precisely using an Ussing chamber. This procedure has been used for the transport of flavonoids in the gastrointestinal mucosa. ${ }^{13)} \mathrm{Hu}$ et al. ${ }^{14)}$ demonstrated that intestinal tissue was viable for $3 \mathrm{~h}$ with an Ussing chamber. We also measured TEER and confirmed the viability of the above.

We found that $\alpha \mathrm{G}$-rutin is transported through the gastric and intestinal mucosa in intact form. Transport of $\alpha$ G-rutin increased time-dependently in the mucosa of the whole alimentary tract, including the stomach. It has been reported in in vivo studies that rutin is scarcely absorbed as such in the stomach or small intestine, is but hydrolyzed by enterobacterial enzymes and absorbed from the colon. ${ }^{12,15,16)}$ In contrast, we found that $\alpha \mathrm{G}$ rutin is transported through the mucosa of the stomach and small intestine. These results suggest that $\alpha \mathrm{G}$-rutin is more efficiently absorbed after oral ingestion than rutin. Furthermore, we found that $\alpha \mathrm{G}$-rutin and rutin 
Table 1. TEER in the Rat Intestinal Epithelium before and after Incubation for $30 \mathrm{~min}$ with $\alpha \mathrm{G}$-Rutin

\begin{tabular}{|c|c|c|c|}
\hline & \multicolumn{3}{|c|}{$\operatorname{TEER}\left(\Omega \mathrm{cm}^{-2}\right)$} \\
\hline & Pre-incubation & $\begin{array}{l}\text { Post-incubation } \\
\text { (100 mM } \alpha \text { G-rutin }\end{array}$ & \\
\hline Stomach fundus & $2,668 \pm 622.0 \mathrm{~b}$ & $2,840 \pm 683.0$ & $\mathrm{~b}$ \\
\hline Stomach body & $163 \pm 14.0 \mathrm{a}$ & $178 \pm 14.0$ & $\mathrm{a}$ \\
\hline Jejunum & $160 \pm 5.4 \mathrm{a}$ & $160 \pm 10.0$ & $\mathrm{a}$ \\
\hline Ileum & $140 \pm$ & $148 \pm 7.3$ & $\mathrm{a}$ \\
\hline Cecum & $170 \pm 11.8 \mathrm{a}$ & $208 \pm 22.0$ & a \\
\hline Colon & $205 \pm 19.0 \mathrm{a}$ & $230 \pm 18.0$ & $\mathrm{a}$ \\
\hline ANOVA $P$-values & & & \\
\hline Portions (P) & \multicolumn{3}{|c|}{$<0.001$} \\
\hline Pre- or post-incubation (I) & \multicolumn{3}{|c|}{0.7823} \\
\hline $\mathrm{P} \times \mathrm{I}$ & \multicolumn{3}{|c|}{0.9995} \\
\hline
\end{tabular}

Values are means $\pm \mathrm{SEM}, \mathrm{n}=6$. Values in two columns not sharing a superscript letter are significantly different $(P<0.05)$ by Duncan's multiple-range test. TEER, transepithelial electrical resistance.

were absorbed in the gastric body but not in the gastric fundus comparably with the other intestines, suggesting that the gastric body might be involved in absorption in the stomach. We observed very high TEER in the mucosa of the gastric fundus. The keratinization of the mucosal surface of the gastric fundus may be involved in the high TEER and low absorptive activities in this segment. The mechanism for flavonoid glycoside absorption in the stomach has not been clarified, and should be in the future.

We found that comparable amounts of rutin and $\alpha \mathrm{G}$ rutin appeared in the serosal fluid in the jejunal and ileal mucosa despite the occurrence of $\alpha \mathrm{G}$-rutin in the cecum and colon. $\alpha \mathrm{G}$-rutin is stable for the analytical procedure used in this study. Its preparation contains less than $1 \%$ rutin (data not shown). We also confirmed that rutin was present in the mucosal fluid of the jejunum and ileum specimens (9.2\% and $16.1 \%$ of $\alpha \mathrm{G}$-rutin respectively). These results suggest that rutin is produced mainly from $\alpha$ G-rutin on the mucosal side of the small intestine. It has been shown that $\beta$-glycosidase in the rat small intestine efficiently hydrolyzes quercetin-3- $O$ - $\beta$-glucosides, whereas rutin is a poor substrate for this enzyme. ${ }^{17,18)}$ Lactose-phlorizin hydrolase (LPH) in the sheep small intestine can also hydrolyze some quercetin glucosides, but not rutin. ${ }^{19)}$ These previous reports agree with our present result that quercetin aglycone was not detected in the mucosal or serosal fluids. The transport pathway responsible for rutin ought to be clarified.

The mechanism by which intact $\alpha \mathrm{G}$-rutin passes through the gastric and intestinal mucosa is not yet known. It has been found that quercetin-3-O-glucoside is absorbed as glycoside into the mucosal cells by way of sodium-dependent glucose transport ${ }^{8,20,21)}$ but it is uncertain whether that $\alpha \mathrm{G}$-rutin, consisting of three sugar moieties, passes through the brush border membrane as it is. There was a linear increase in $\alpha$ G-rutin transport from the mucosal side to the serosal side in the three intestinal segments at concentrations up to $100 \mathrm{~mm}$ $\alpha$ G-rutin (Fig. 4). This unsaturable increase in transport suggests that a simple diffusion is responsible for $\alpha \mathrm{G}$ rutin absorption. It is more likely that $\alpha \mathrm{G}$-rutin is transported via tight junctions of the intestinal mucosa by diffusion. ${ }^{13)}$ Morand et $a l .{ }^{6)}$ showed that quercetin was conjugated with glucuronide in the intestinal cells. After ingestion of quercetin aglycone or quercetin-3-Oglucoside, they are absorbed and conjugated in the intestinal cells. Absorption of $\alpha$ G-rutin through the paracellular pathway might be associated with no detection of quercetin aglycone or its conjugate in the serosal fluid. The difference in metabolism between these two glycosides might be due to differences in the absorptive pathways.

We conclude that a considerable portion of $\alpha \mathrm{G}$-rutin was absorbed intact across the mucosa of the stomach and intestine and was in part hydrolyzed to rutin on the mucosal side of the small intestine.

\section{References}

1) Sakakibara, H., Honda, Y., Nakagawa, S., Ashida, H., and Kanagawa, K., Simultaneous determination of all polyphenols in vegetables, fruits, and teas. J. Agric. Food Chem., 51, 571-581 (2003).

2) Vinson, J. A., Su, X., Zubik, L., and Bose, P., Phenol antioxidant quantity and quality in foods: fruits. J. Agric. Food Chem., 49, 5315-5321 (2001).

3) O'Reilly, J. D., Mallet, A. I., McAnlis, G. T., Young, I. S., Halliwell, B., Sanders, T. A., and Wiseman, H., Consumption of flavonoids in onions and black tea: lack of effect on F2-isoprostanes and autoantibodies to oxidized LDL in healthy humans. Am. J. Clin. Nutr., 73, 1040-1044 (2001).

4) Rechner, A. R., Wagner, E., Van Buren, L., Van De Put, F., Wiseman, S., and Rice-Evans, C. A., Black tea represents a major source of dietary phenolics among regular tea drinkers. Free Radic. Res., 36, 1127-1135 (2002).

5) Liu, I. M., and Sheu, S. J., Analysis and processing of Chinese herbal drugs. VIII: The study of sophorae floe. Am. J. Chin. Med., 17, 179-187 (1989).

6) Morand, C., Manach, C., Crespy, V., and Remesy, C., Quercetin 3-O- $\beta$-glucoside is better absorbed than other quercetin forms and is not present in plasma. Free Radic. Biol. Med., 33, 667-676 (2000).

7) Crespy, V., Morand, C., Besson, C., Manach, C., Demigne, C., and Remesy, C., Comparison of the intestinal absorption of quercetin, phloretin and their glucosides in rats. J. Nutr., 131, 2109-2114 (2001).

8) Wolffram, S., Block, M., and Ader, P., Quercetin-3glucoside is transported by the glucose carrier SGLT1 across the brush border membrane of rat small intestine. J. Nutr., 132, 630-635 (2002).

9) Hollman, P. C., de Vries, J. H., van Leeuwen, S. D., Mengelers, M. J., and Katan, M. B., Absorption of dietary quercetin glucosides and quercetin in healthy ileostomy volunteers. Am. J. Clin. Nutr., 62, 1276-1282 (1995).

10) Manach, C., Morand, C., Texier, O., Favier, M. L., Agullo, G., Demigne, C., Regerat, F., and Remesy, C., Quercetin metabolites in plasma of rats fed diets 
containing rutin or quercetin. J. Nutr., 125, 1911-1922 (1995).

11) Hollman, P. C., Bijsman, M. N., van Gameren, Y., Cnossen, E. P., de Vries, J. H., and Katan, M. B., The sugar moiety is a major determinate of the absorption of dietary flavonoid glycosides in man. Free Radic. Res., 31, 569-573 (1999).

12) Crespy, V., Morand, C., Manach, C., Besson, C., Demigne, C., and Remesy, C., Part of quercetin absorbed in the small intestine is conjugated and further secreted in the intestinal lumen. Am. J. Physiol., 277, 120-126 (1999).

13) Mineo, H., Hara, H., Shigematsu, N., Okuhara, Y., and Tomita, F., Melibiose, difructose anhydride III and difructose anhydoride IV enhance net calcium absorption in rat small and large intestinal epithelium by increasing the passage of tight junctions in vitro. J. Nutr., 132, 3394-3399 (2002).

14) Hu, M. S., Kayne, L. H., Willsey, P. A., Koteva, A. B., Jamgotchian, N., and Lee, D. B., Bile salts and ileal calcium transport in rats: a neglected factor in intestinal calcium absorption. Am. J. Physiol., 264, 319-324 (1993).

15) Baba, S., Furuta, T., Fujioka, M., and Goromaru, T., Studies on drug metabolism by use of isotopes XXVII: urinary metabolites of rutin in rats and the role of intestinal microflora in the metabolism of rutin. $J$. Pharm. Sci., 72, 1155-1158 (1983).
16) Crespy, V., Morand, C., Besson, C., Manach, C., Demigne, C., and Remesy, C., Quercetin, but not its glycosides, is absorbed from the rat stomach. J. Agric. Food Chem., 50, 618-621 (2002).

17) Ioku, K., Pongpiriyadacha, Y., Konishi, Y., Takei, Y., Nakatani, N., and Terano, J., $\beta$-glucosidase activity in the rat small intestine toward quercetin monoglucosides. Biosci. Biotechnol. Biochem., 62, 1428-1431 (1998).

18) Day, A. J., Dopont, M. S., Ridley, S., Rhodes, M., Rhodes, M. J. C., Morgan, M. R. A., and Williamson, G., Deglycosylation of flavonoid and isoflavonoid glycosides by human small intestine and liver $\beta$-glucosidase activity. FEBS Lett., 436, 71-75 (1998).

19) Day, A. J., Canada, F. J., Diaz, J. C., Kroon, P. A., Mclauchlan, R., Faulds, C. B., Plumb, G. W., Morgan, M. R., and Williamson, G., Dietary flavonoid and isoflavone glycosides are hydrolysed by the lactase site of lactase phlorizin hydrolase. FEBS Lett., 468, 166-170 (2000).

20) Walgren, R. A., Walle, U. K., and Walle, T., Transport of quercetin and its glucosides across human intestinal epithelial Caco-2 cells. Biochem. Pharmacol., 55, 17211727 (1998).

21) Gee, J. M., DuPont, M. S., Rhodes, M. J., and Jhonson, I. T., Quercetin glucosides interact with the intestinal glucose transport pathway. Free Radic. Biol. Med., 25, 19-25 (1998). 